\title{
RESEARCH
}

Open Access

\section{YBX-1 mediated sorting of miR-133 into hypoxia/reoxygenation-induced EPC- derived exosomes to increase fibroblast angiogenesis and MEndoT}

Fengxia Lin ${ }^{1}$, Zhicong Zeng ${ }^{1}$, Yinzhi Song ${ }^{1}$, Liang Li, ${ }^{1,2}$, Zijun Wu ${ }^{1}$, Xiaoduo Zhang ${ }^{1}$, Zhiwen $\mathrm{Li}^{1}$, Xiao Ke ${ }^{3,4^{*}}$ and Xun $\mathrm{Hu}^{5,6^{*}}$

\begin{abstract}
Background: Myocardial fibrosis is a common pathophysiological change in cardiovascular disease, which can cause cardiac dysfunction and even sudden death. Excessively activated fibroblasts proliferate and secret excessive extracellular matrix (ECM) components, resulting in normal cardiac structural damage and cardiac fibrosis. We previously found that human endothelial progenitor cell (EPC)-derived exosomes, after hypoxia/reoxygenation (H/R) induction, could significantly increase the mesenchymal-endothelial transition (MEndoT) compared to normal culture EPC-derived exosomes. Exosomes have been shown to carry different nucleic acids, including microRNAs. However, the effects of microRNAs in EPC-derived exosomes on MEndoT and myocardial fibrosis remain unknown.

Methods: EPCs were isolated from human peripheral blood, and fibroblasts were isolated from rat hearts, then transfected with miR-133 inhibitor, si-YBX-1, and ov-YBX-1 into EPCs. After H/R induction for $48 \mathrm{~h}$, isolation and characterization of exosomes derived from human EPCs were performed. Finally, fibroblasts were treated by exosome at $48 \mathrm{~h}$. The expression of miR-133 was measured by qRT-PCR; YBX-1 expression was measured by qRT-PCR and western blot. Angiopoiesis was measured by tube formation assay. Endothelial markers and fibrosis markers were measured by western blot.

Results: H/R treatment promoted miR-133 expression in EPCs and EPC-derived exosomes. miR-133 could be incorporated into exosomes and transmitted to cardiac fibroblasts, increasing the angiogenesis and MEndoT of cardiac fibroblasts. miR-133 silencing in H/R-induced EPCs could inhibit miR-133 expression in EPCs and EPCsderived exosomes. miR-133 silencing in H/R-induced EPCS could inhibit the angiogenesis and MEndoT of cardiac fibroblasts and reverse the effect of $\mathrm{H} / \mathrm{R}$ treatment. Additionally, miR-133 was specially sorted into H/R-induced EPCderived exosomes via YBX-1. YBX-1 silencing inhibited miR-133 transfer and reduced fibroblast angiogenesis and MEndoT.
\end{abstract}

Conclusion: miR-133 was specially sorted into H/R-induced EPC-derived exosomes via YBX-1 to increase fibroblast angiogenesis and MEndoT.

Keywords: Endothelial progenitor cell, Myocardial fibrosis, miR-133, Exosome, Y box binding protein 1

\footnotetext{
* Correspondence: kexiao@email.szu.edu.cn; huxunsysu@163.com

${ }^{3}$ Department of Cardiology, Fuwai Hospital, Chinese Academy of Medical

Sciences, Shenzhen 518057, Guangdong, China

${ }^{5}$ Department of Cardiology, The First Affiliated Hospital, Sun Yat-Sen

University, Guangzhou 510080, Guangdong, China

Full list of author information is available at the end of the article
}

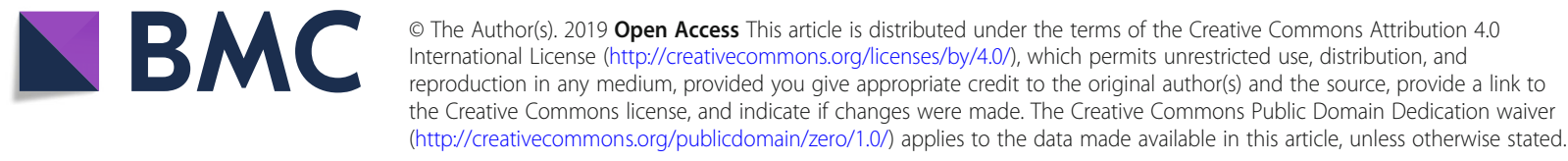




\section{Background}

Myocardial fibrosis is a common pathophysiological change in cardiovascular disease, which can cause cardiac dysfunction and even sudden death [1]. It is one of the important causes of death in patients with cardiovascular disease. Therefore, reversing myocardial fibrosis is an important goal in the treatment of cardiovascular disease. Fibroblasts are key effector molecules of cardiac fibrosis [2]. Cardiac fibroblasts play an important role in maintaining cardiac structure and function by synthesizing collagen I, collagen III, fibronectin, and other extracellular matrix components. Under pathological conditions, fibroblasts proliferate and activate excessive secretion of extracellular matrix components, resulting in normal cardiac structural damage and cardiac fibrosis. Cardiac fibroblasts were once thought to be terminally differentiated cells. However, recent studies have shown that chromatin-modifying agents could activate Wnt signaling to transform human adult dermal fibroblast cells to OCT4+ and VEGFR-2+ capillary tube-forming cells [3]. When pathological damage occurs in the heart, fibroblasts undergo a mesenchymal-endothelial transition (MEndoT) to obtain endothelial cell-like functions and participate in angiogenesis in the cardiac injury area, which is a novel antifibrotic strategy to alleviate myocardial fibrosis [4]. However, the mechanism underlying the transformation of cardiac fibroblasts into endothelial cells during pathological heart damage remains unclear.

Previously, we found that hypoxia/reoxygenation (H/R)-induced human endothelial progenitor cell (EPC)-derived exosomes increased proliferation and angiogenesis of cardiac fibroblasts by promoting MEndoT [5]. Exosomes are small membrane vesicles that mediate intercellular signal transduction [6]. Previous studies have shown that EPC-derived exosomes can promote angiogenesis and reduce cardiomyocyte hypertrophy and apoptosis [7-9]. Furthermore, exosomes had been shown to carry different nucleic acids, including microRNAs (miRNAs), which are acquired by recipient cells to regulate their own fate $[10,11]$. Previous studies found that miRNAs in exosomes from endothelial progenitor cells improved outcomes of patients with cardiovascular disease [12] and lipopolysaccharide-induced acute lung injury [13] and also of a murine model of sepsis [12].

miRNAs significantly regulate cell growth and metabolism through post-transcriptional inhibition of gene expression and play important roles in myocardial fibrosis [14-16]. miR-181a and miRNA-221/222 regulated cardiac fibroblast activation to increase deposition of extracellular matrix components and promote myocardial fibrosis [17, 18]. miR-378 in exosomes, which is secreted from cardiomyocytes, inhibited excessive cardiac fibrosis [19]. Further, miR-217 in exosomes, which is secreted from cardiomyocytes, promoted cardiac fibrosis processes, cardiac hypertrophy, and cardiac fibrosis processes [20]. However, the effects of miRNA in EPC-derived exosomes on MEndoT and myocardial fibrosis remain unknown.

In our previous study, we found that EPC-derived exosomes, after $H / R$ induction, could significantly increase MEndoT compared with normal culture EPC-derived exosomes [5]. In this study, we collected exosomes derived from normal and H/R-cultured EPCs and measured miRNA expression using a miRNA array. Additionally, we investigated the effect of miRNAs in EPC-derived exosomes on angiogenesis and MEndoT. Finally, we examined the mechanism of miRNA assembly into exosomes.

\section{Materials and methods}

Isolation, identification, and culture of EPCs and fibroblasts EPCs were isolated from human peripheral blood, and fibroblasts were isolated from rat hearts which are identified by immunofluorescence and flow cytometry as previously described [5]. Isolated EPC cells were placed into a $25-\mathrm{cm}^{2}$ culture bottle and cultured in Dulbecco's modified Eagle's medium (DMEM; Gibco) containing $10 \%$ fetal bovine serum, which was centrifuged in advance by density gradient centrifugation to remove existing exosomes (Gibco), in a $5 \% \mathrm{CO}_{2}$ humidified environment at $37^{\circ} \mathrm{C}$. The medium was changed every 3 days. Late passage cells (p3) were used in subsequent experiments. Isolated fibroblasts were suspended in DMEM (Gibco) with 10\% FBS (Gibco). After $30 \mathrm{~min}$, cells that were weakly attached or unattached were discarded. Cells were seeded onto $35-\mathrm{mm}$ plates $\left(1 \times 10^{5}\right.$ cells/plate) for 3 days.

\section{$\mathrm{H} / \mathrm{R}$ treatment}

EPCs were grown to $80 \%$ confluence and quiesced for $12 \mathrm{~h}$. Plated cells were subjected to normoxic or hypoxic conditions for $12 \mathrm{~h}$. To generate hypoxic conditions, cells were transferred to an incubation chamber (Billups-Rothenberg MIC-101) and flushed with hypoxic gas mixture $\left(95 \% \mathrm{~N}_{2}, 5 \% \mathrm{CO}_{2}\right)$. Subsequently, the cells were cultured in normoxic conditions for $48 \mathrm{~h}$.

\section{Isolation and characterization of exosomes derived from human EPCs}

Exosomes derived from H/R-treated EPCs were isolated and identified as previously described [5]. Following H/R treatment of EPCs, the original medium was replaced with fresh exosomal-free serum medium, and the cells were cultured for $24 \mathrm{~h}$. Culture media of EPCs were collected and centrifuged at $3000 \mathrm{~g}$ for $30 \mathrm{~min}$ and 100,000 $g$ for $90 \mathrm{~min}$ at $4{ }^{\circ} \mathrm{C}$ to remove dead cells and cellular 
debris by Optima Ultracentrifuge (Beckman Coulter). The medium was mixed with $0.5 \mathrm{~mL}$ of Total Exosome Isolation reagent (GENESEED, Guangzhou, China), centrifuged at $10,000 \mathrm{~g}$ for $1 \mathrm{~h}$ at $4{ }^{\circ} \mathrm{C}$ to obtain exosomes. Exosome morphology was visualized using a transmission electron microscope (Hitachi H-7650; Japan), and images were taken with a digital camera (Olympus). Surface proteins (CD63, TSG101, and HSP70) on the exosomes were detected by western blotting. Finally, EPC-derived exosomes were added to the fibroblast culture medium.

\section{Apoptosis and senescence assay}

The apoptosis of H/R-treated EPCs was measured by the Annexin V-fluorescein isothiocyanate (FITC) Apoptosis Detection Kit (Keygentec, Nangjing, China). The senescence of H/R-treated EPCs was measured by Senescence $\beta$-galactosidase staining kit (Beyotime, Shanghai, China).

\section{miRNA profiling}

Exosomal RNA was extracted using the TRIzol reagent (Life Technologies, Carlsbad, CA, USA). miRNA expression profile in exosomes was investigated by miRNA microarray analysis. Exosomal miRNAs were extended and hybridized with fluorescent-labeled biotin dyes on a Gene Chip miRNA 4.0 Array (Affymetrix, Cleveland, $\mathrm{OH}$, USA). Following hybridization, the images were digitized and analyzed using a laser scanner interfaced with ArrayPro image analysis software (Media Cybernetics, Silver Spring, MD, USA). Data were analyzed by first subtracting the background, followed by normalizing the signals using a LOWESS filter (locally weighted regression) [21]. The differentially expressed miRNAs were defined using the ratio of detected signals $\log 2$-fold changes $[\log 2(\mathrm{mTLE}-\mathrm{HS} / \mathrm{control})]$, and the Student's $t$ test was used to calculate $P$ values. Those with a $\log 2$ ratio $>1.0$ or $\leq-1.0$ and $P$ values $<0.05$ were considered as differentially expressed miRNAs. Cluster analysis

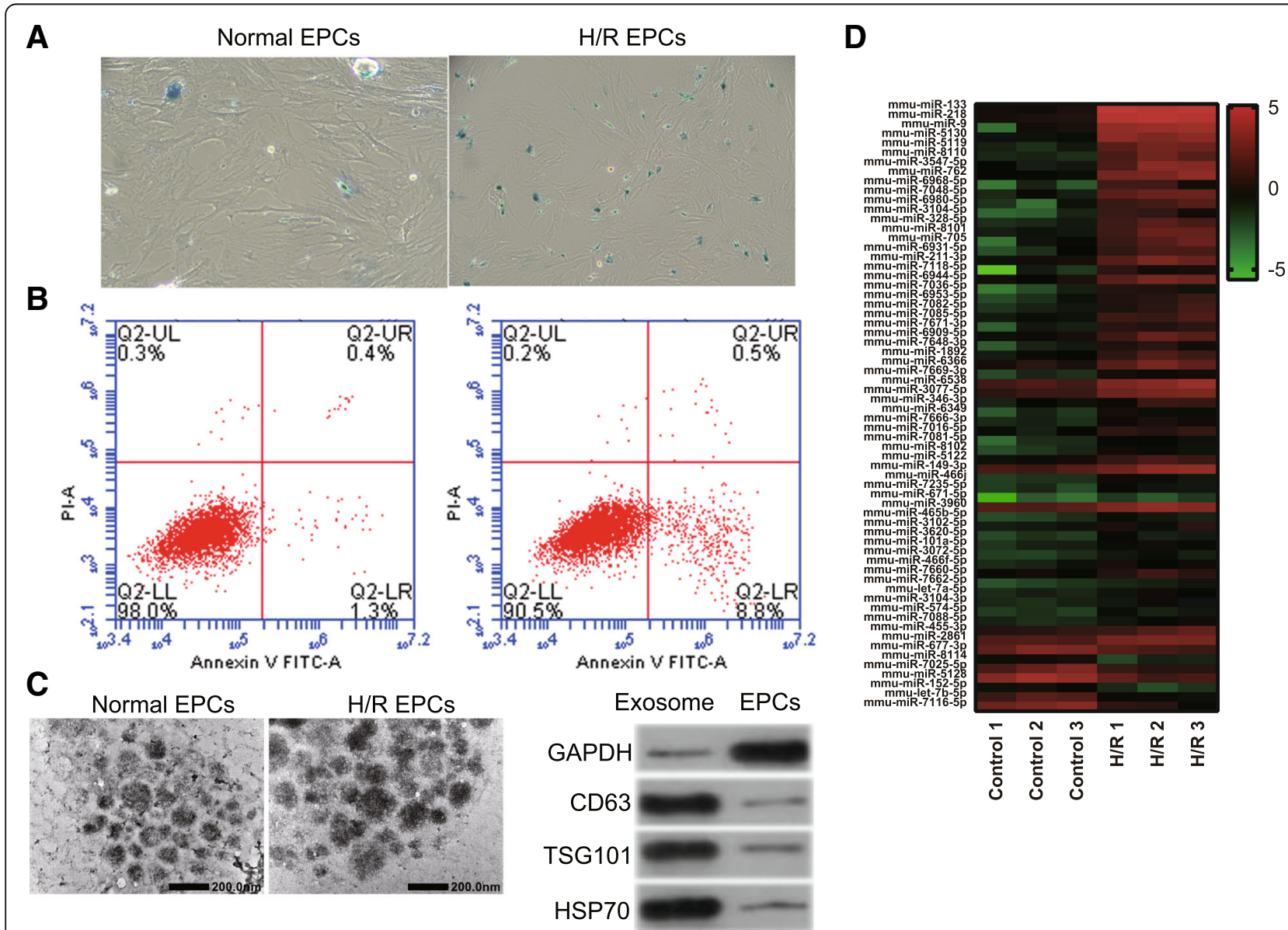

Fig. 1 miRNA expression in H/R-induced EPC-derived exosomes. a Senescence of H/R-treated EPCs was measured by senescence $\beta$-galactosidase staining kit. Senescence cells were stained aquamarine green. $\mathbf{b}$ Apoptosis of H/R-treated EPCS was measured by the Annexin V-PI Apoptosis Detection Kit. c Representative electron microscopy images of exosomes secreted by EPCs. Scale bar, $200 \mathrm{~nm}$. Detection of exosome-associated proteins (including CD63, TSG101, and HSP70) by western blot analysis. $\mathbf{d}$ Heat map showing the miRNA expression profile as measured by Affymetrix miRNA 4.0 Arrays 

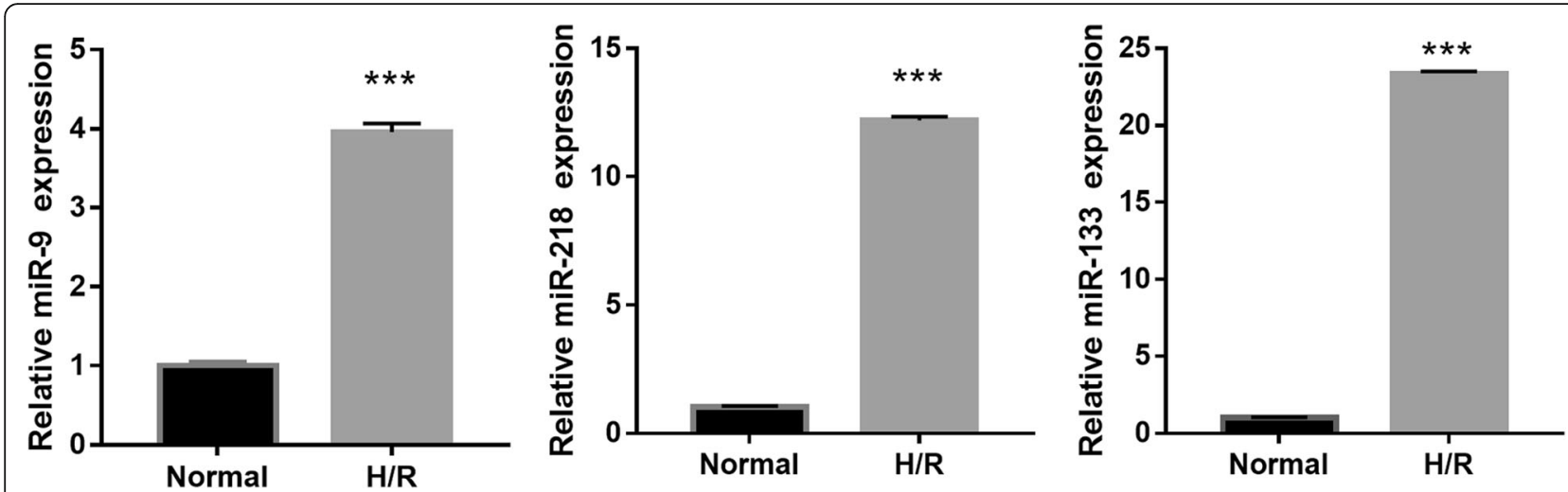

Fig. 2 Upregulation of miR-133, miR-218, and miR-9 expression in H/R-induced EPC-derived exosomes. Expression levels of miR-133, miR-218, and miR-9 were measured by qRT-PCR in H/R-induced and normal cultured EPC-derived exosomes. Data are shown as mean \pm SD. ${ }^{* * *} P<0.001$

based on the relative expression levels of miRNAs was also conducted.

\section{Quantitative real-time reverse transcription-polymerase chain reaction}

Total RNA was isolated using the TRIzol reagent (Invitrogen, Carlsbad, CA, USA) and reverse transcribed. qRT-PCR was performed using the SYBR $^{\circ}$ Premix ExTaqTM II Kit (Takara, Dalian, China) to detect YBX1 expression and the Mir-X miRNA qRT-PCR SYBR Kit (Clontech Laboratories, Inc., USA) to detect miR-133 on a 7500 Real-Time PCR System (Applied Biosystems, Foster City, CA, USA). The relative expression levels of mRNA and miRNA were calculated using the 2- $\Delta \Delta C \mathrm{CT}$ method. GAPDH and U6 served as reference genes, respectively. The primer sequences were as follows: YBX-1 forward, $5^{\prime}$-GATAAATTTAAACCTGAAAA- 3 ' and reverse, 5'-ATCTTGTTTCTATCTTCCAA-3'; miR-133 forward, 5' - ACACTCCAGCTGGGCAAAGTGCTTAC AGTGC-3' and reverse, 5'-CTCAACTGGTGTCG TGGA-3'; U6 forward, 5'-CTCGCTTCGGCAGCACA$3^{\prime}$ and reverse, 5'-AACGCTTCACGAATTTGCGT-3'; GAPDH forward, 5'-GCTCATTTGCAGGGGGGAG-3' and reverse, 5'-GTTGGTGGTGCAGGAG GCA-3'. All reactions were performed in triplicate.

\section{Transfection}

YBX-1 expression interference (si-YBX-1), si-negative control (si-NC), miR-133 inhibitor, negative control inhibitor (NC inhibitor), miR-133 mimic, and NC mimic were purchased from RiboBio (Guangzhou, China). The open reading frame of YBX was synthesized and linked
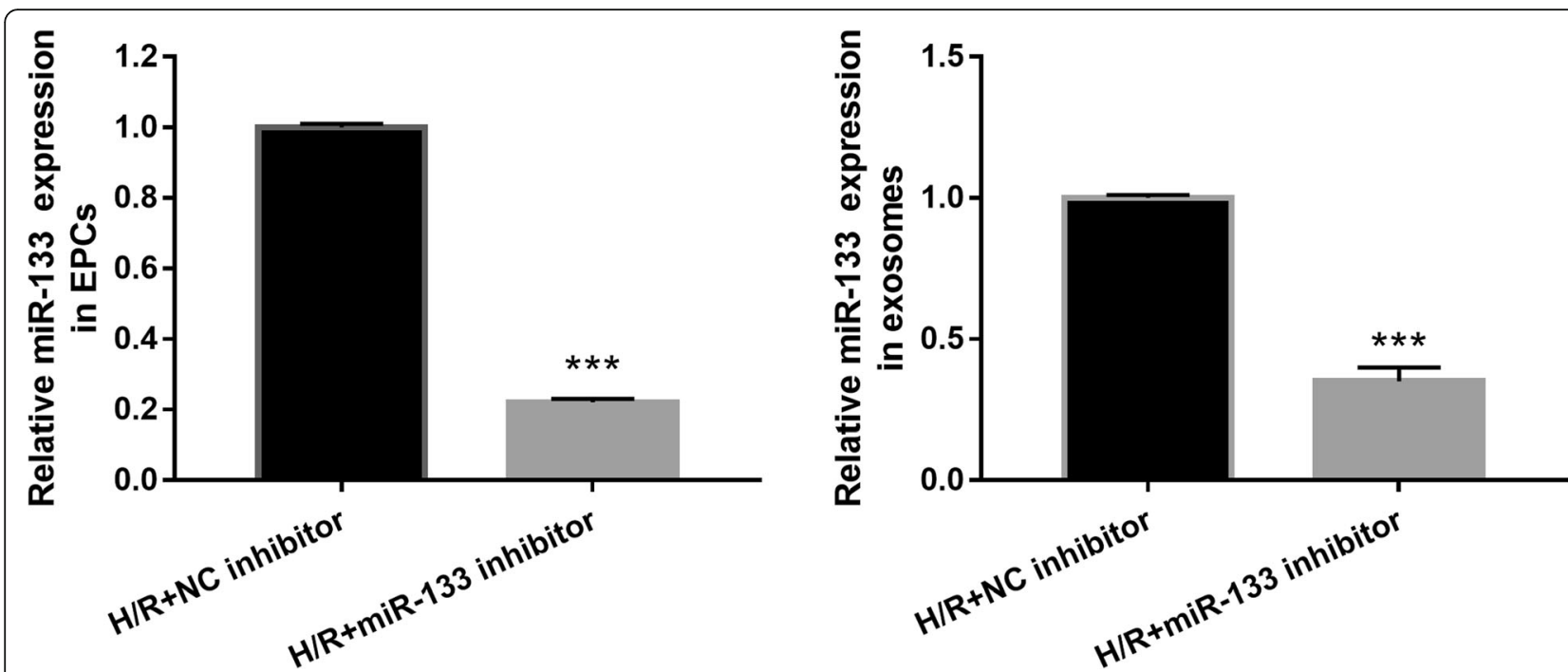

Fig. 3 Significant inhibition of miR-133 expression by miR-133 inhibitor transfection in H/R-induced EPCs and H/R-induced EPC-derived exosomes. miR-133 expression was measured by qRT-PCR after transfection with an NC inhibitor and miR-133 inhibitor at $48 \mathrm{~h}$. Data are shown as mean \pm SD. ${ }^{* * *} P<0.001$ 
into pcDNA 3.1 (ov-YBX-1), and pcDNA 3.1 served as a negative control (ov-NC). EPCs $\left(2 \times 10^{5}\right.$ cells/well) were transfected with $50 \mathrm{nM}$ miR-133 inhibitor, $50 \mathrm{nM}$ $\mathrm{NC}$ inhibitor, $50 \mathrm{nM}$ si-YBX-1, $50 \mathrm{nM}$ si-NC, $1 \mu \mathrm{g} / \mu \mathrm{L}$ ov-YBX-1, and $1 \mu \mathrm{g} / \mathrm{uL}$ ov-NC using Lipofectamine ${ }^{\mathrm{mm}}$ 2000 (Invitrogen) according to the manufacturer's instructions.

\section{Tube formation assay}

Matrigel $(300 \mathrm{~mL}$ per well) was plated onto the bottom of six-well plates and incubated at $37^{\circ} \mathrm{C}$ for 30 min. Fibroblasts $\left(1 \times 10^{5}\right.$ cells per well $)$ were seeded on Matrigel and induced by EPC-derived exosomes. After a 48-h culture, tube formation was assessed using an inverted microscope (Olympus, Tokyo, Japan).

\section{Western blotting assay}

Western blotting was performed to analyze the expression of CD31, $\alpha$-SMA, VE-cadherin, vWF, N-cadherin, vimentin, collagen I, YBX-1, SYNCRIP, and hnRNPA2B1 [5]. Specific primary antibodies against CD31 (MA181051, 1:100), $\alpha$-SMA (14-9760-82, 1:500), VE-cadherin (MA5-17050, 1:500), vWF (PA5-80223, 1:1000), N-cadherin (33-3900, 1:1000), vimentin (MA3-745, 1:1000), collagen I (PA1-26204, 1:5000), YBX-1 (PA5-83493, 1:100), SYNCRIP (PA5-50986, 1:500), and hnRNPA2B1 (PA534939, 1:5000) were purchased from eBioscience (San Diego, CA, USA), and an HRP-conjugated goat anti-rabbit IgG H\&L secondary antibody (1:10000; Southern Biotech, Birmingham, AL, USA) was used. Protein bands were visualized using ECL (Thermo Fisher Scientific). Protein expression was normalized relative to GAPDH expression.

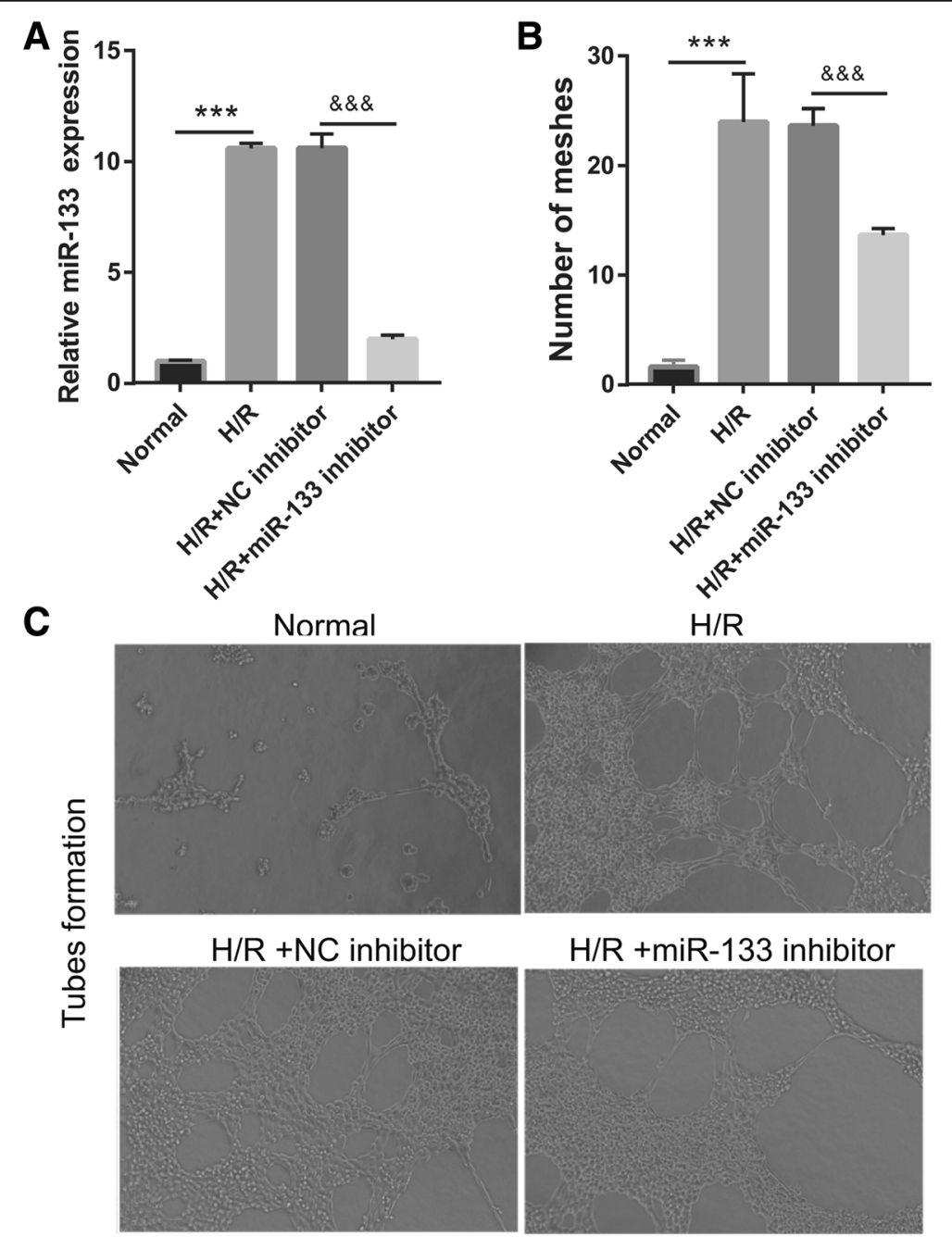

Fig. 4 Silencing of miR-133 in H/R-induced EPCs inhibits fibroblast angiogenesis. a miR-133 expression was measured by qRT-PCR in fibroblasts treated with EPC-derived exosomes for $48 \mathrm{~h}$. $\mathbf{b}$ The bar graph represents quantification of the number of meshes per group. $\mathbf{c}$ Representative image of tube formation analysis. Data are shown as mean \pm SD. ${ }^{* *} P<0.001,{ }^{\& \& \&} P<0.001$ 


\section{Results}

miR-133 expression is upregulated in H/R-induced EPC (H/R-EPC)-derived exosomes (H/R-EPC-exosomes)

First, we found that the senescence and apoptosis in $\mathrm{H} /$ R-induced EPC were obviously enhanced compared to normal cultured EPCs (Fig. 1a, b). Then, exosomes were purified from culture medium and confirmed by transmission electron microscopy and exosome markers CD63, TSG101, and HSP70 (Fig. 1c). Additionally, miRNA profiles of exosomes from $\mathrm{H} / \mathrm{R}$-induced and normal cultured EPCs were investigated using Affymetrix miRNA 4.0 Arrays. The microarray results showed differential expression of 64 miRNAs (7 downregulated miRNAs and 57 upregulated miRNAs, log-fold change $>1 ; P<0.05)$ in $\mathrm{H} / \mathrm{R}$-EPC-exosomes compared to normal cultured EPC-derived exosomes (NormalEPC-exosomes) (Fig. 1d). Among 64 miRNAs, only three miRNAs, including miR-133, miR-218, and miR9, were expressed at greater than five times log-fold change (log-fold change $>5 ; P<0.05$ ). To validate the accuracy of the microarray assay, expression levels of miR-133, miR-218, and miR-9 were measured by qRT-PCR in H/R-EPC-exosomes and Normal-EPCexosomes. The results showed that the expression

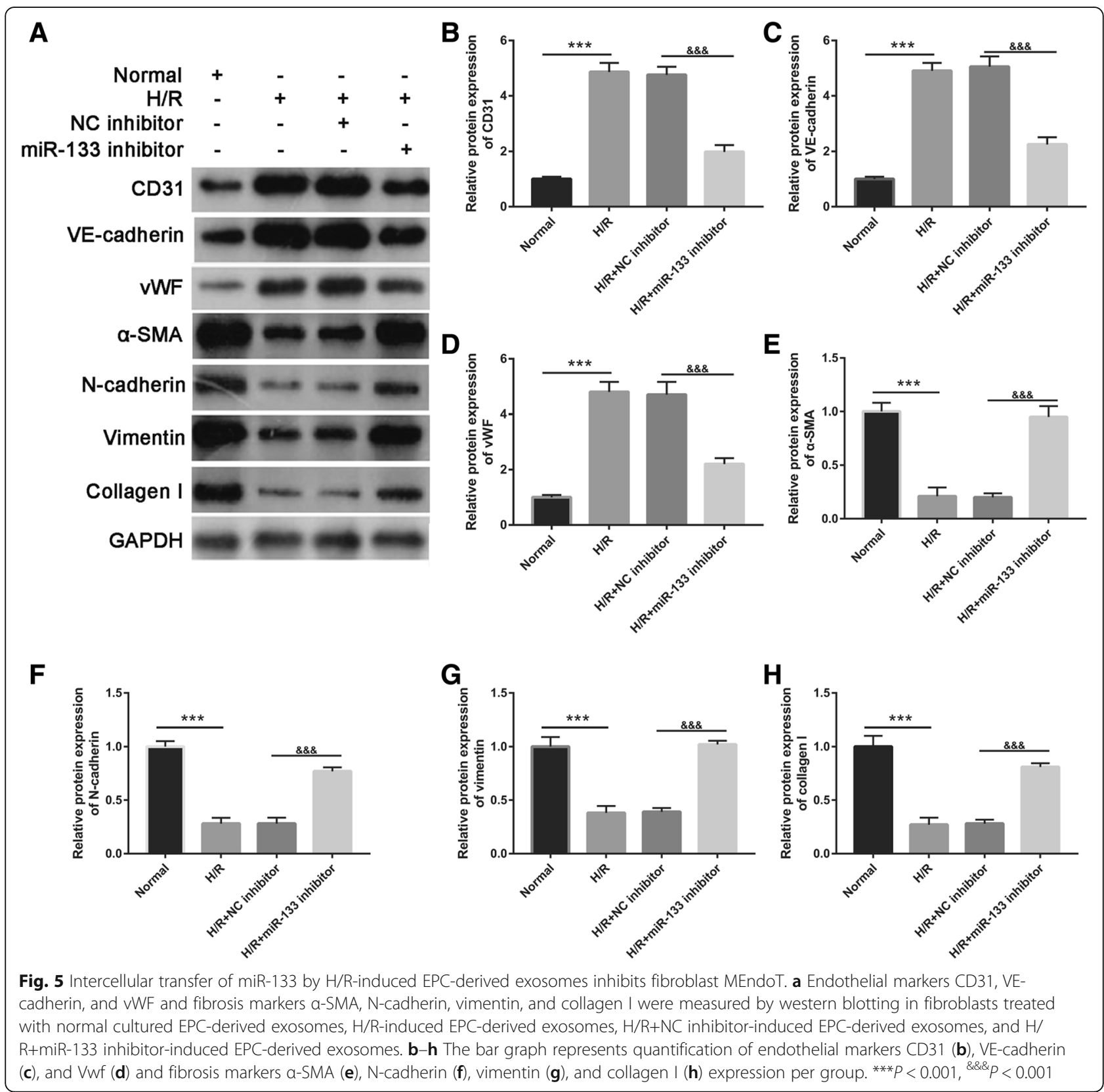


levels of miR-218, miR-9, and especially miR-133 were significantly upregulated in H/R-EPC-exosomes compared to Normal-EPC-exosomes (Fig. 2). Therefore, we focused on miR-133 for further experiments because miR-133 expression had more significant change than miR-218 and miR-9 expression in H/R-EPC-exosomes.

\section{Intercellular transfer of miR-133 by H/R-EPC-exosomes inhibits fibroblast angiogenesis and MEndoT}

In an attempt to identify miR-133 required for fibroblast angiogenesis and MEndoT, the miR-133 inhibitor was transfected into H/R-EPC (H/R- miR-133 inhibitor/EPC). The qRT-PCR results showed that miR-133 expression was significantly inhibited after transfection of the miR-133 inhibitor at $48 \mathrm{~h}$ in H/REPC and H/R-EPC-exosomes compared to NC inhibitor transfection (Fig. 3). Additionally, fibroblasts were treated with H/R-miR-133 inhibitor/EPC-exosomes for $48 \mathrm{~h}$. In the following, angiogenesis and MEndoT of fibroblasts were measured. First, miR-133 expression in fibroblasts treated with Normal-EPC-exosomes was significantly lower than that in fibroblasts treated with H/R-EPC-exosomes and H/R-NC inhibitor/EPC-exosomes. Further, miR-133 expression in fibroblasts treated with H/R-NC inhibitor/EPC-exosomes was significantly higher than that in fibroblasts treated with H/R-miR-133 inhibitor/EPC-exosomes (Fig. 4a). Second, the number of meshes had a similar trend of miR-133 expression in each group, which showed upregulation and downregulation of the number of meshes by miR-133 overexpression and miR-133 knockdown, respectively (Fig. 4b, c). Finally, expression of endothelial markers CD31, VE-cadherin, and vWF in fibroblasts treated with H/R-EPC-exosomes and H/R-NC inhibitor/EPC-exosomes was significantly higher than that in fibroblasts treated with NormalEPC-exosomes and H/R-miR-133 inhibitor/EPC-exosomes. Conversely, fibrosis markers $\alpha$-SMA, N-cadherin, vimentin, and collagen I were significantly lower (Fig. 5). The results showed that intercellular transfer of miR-133 by H/R-EPC-exosomes promoted fibroblast angiogenesis and MEndoT.
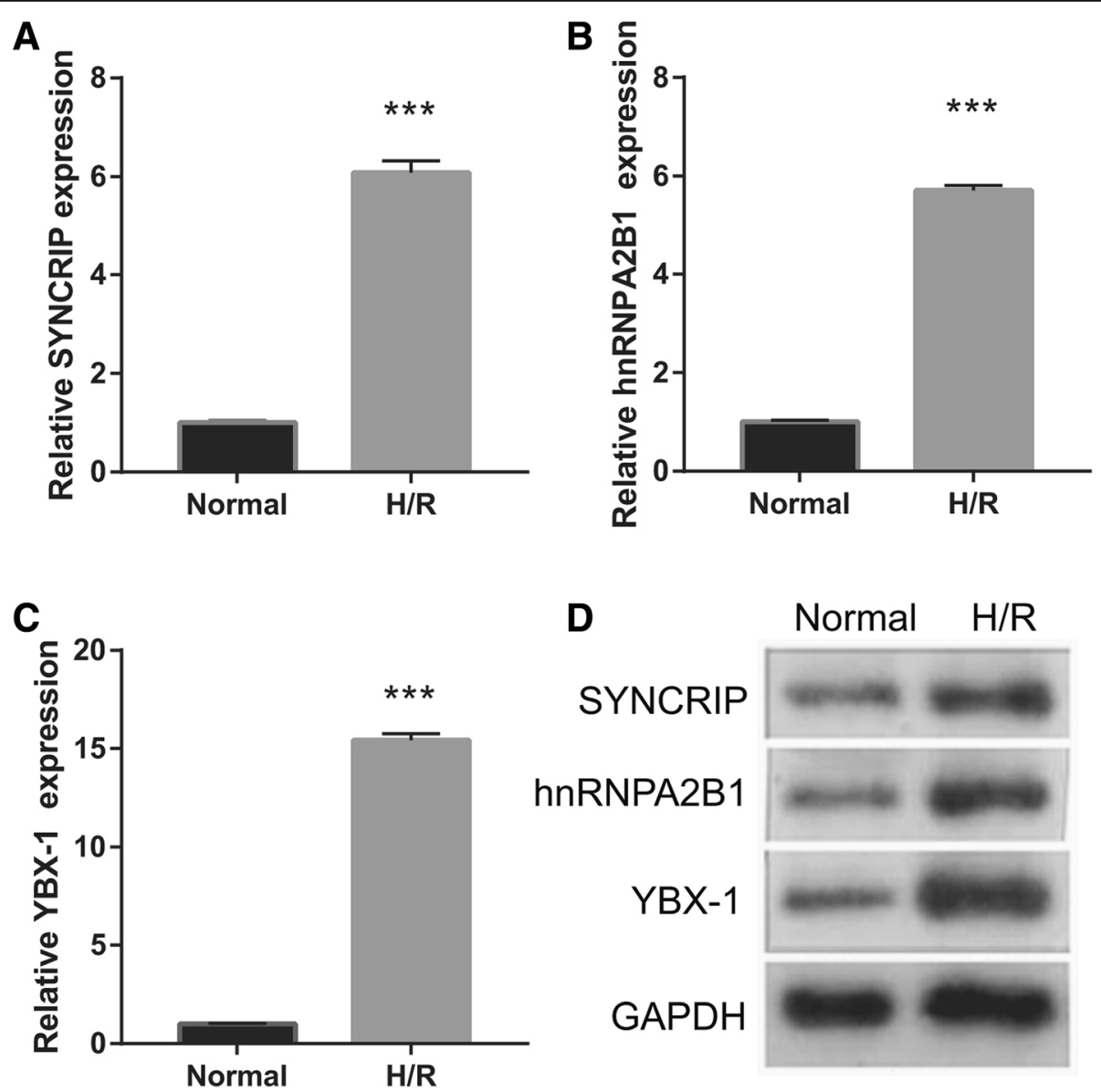

Fig. 6 Measurements of YBX-1, SYNCRIP, and hnRNPA2B1 in EPCs by qRT-PCR (a-c) and western blotting (d) after 48-h H/R treatment 
YBX-1 expression is upregulated in H/R-EPC

We next examined the specific packaging of miR-133 into EPC-derived exosomes, and three reported sorting protein of exosomes, including YBX-1, SYNC RIP, and hnRNPA2B1, in EPCs were measured by qRT-PCR and western blotting. The results showed that YBX-1, SYNCRIP, and hnRNPA2B1 expression levels, especially YBX-1, were significantly upregulated in H/R-EPC compared to Normal-EPC (Fig. 6). Therefore, we focused on YBX-1 for further experiments.

\section{Packaging of miR-133 into EPC-derived exosomes is mediated by YBP1}

Additionally, we silenced YBX-1 by si-YBX1 transfection to study whether miR-133 was specifically packaged into exosomes by YBP1. YBX1 expression was successfully reduced by si-YBX1 transfection in H/R-EPC (Fig. 7a). As expected, knockdown of YBX1 in H/R-EPC significantly decreased miR-133 expression in H/R-EPC- exosomes; however, it did not change miR-133 expression in H/R-EPC (Fig. 7b). Co-transfected si-YBX1 and miR-133 mimic significantly increased miR-133 expression in H/R-EPC with no effect on miR-133 expression in $\mathrm{H} / \mathrm{R}$-EPC-exosomes (Fig. 7c). Next, we upregulated YBX-1 expression by ov-YBX1 transfection to further study whether miR-133 was specifically packaged into exosomes by YBP1. YBX1 expression was successfully enhanced by ov-YBX1 transfection in H/R-EPC (Fig. 8a). As expected, YBX1 overexpression in H/R-EPC had no significant changes in miR-133 expression in H/R-EPC and H/R-EPC-exosomes (Fig. 8b). Co-transfected ovYBX1 and miR-133 mimic significantly increased miR133 expression in H/R-EPC and H/R-EPC-exosomes (Fig. 8c). Additionally, ov-YBX-1 transfected had no significant change in the miR-133 expression in the $\mathrm{H} /$ R-miR-133 inhibitor-treated EPC cells and exosomes compared to ov-NC transfected (Fig. 8d). These results showed that YBP1 mediated miR-133 packaging into EPC-derived exosomes.
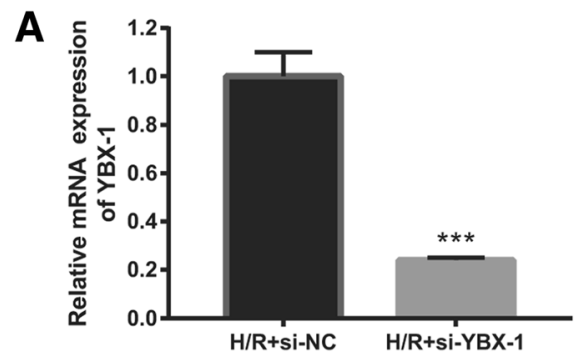

B
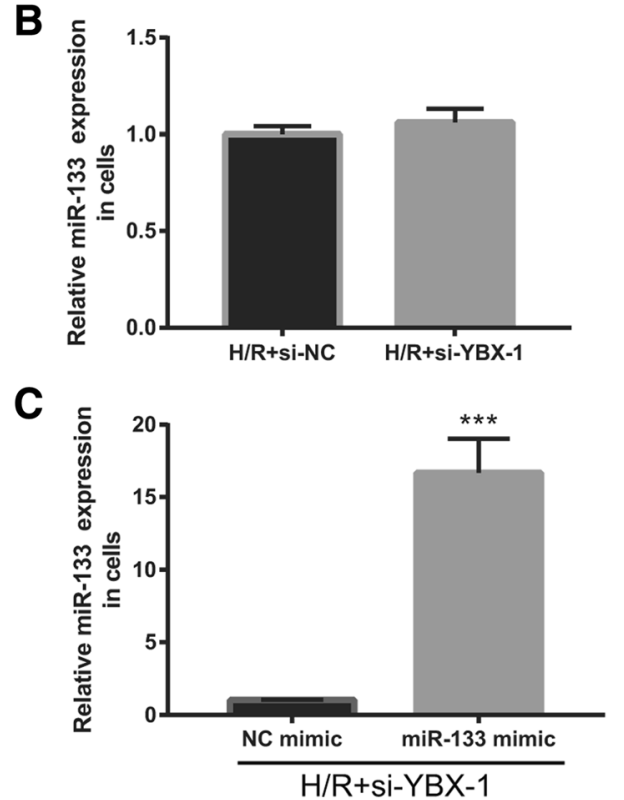
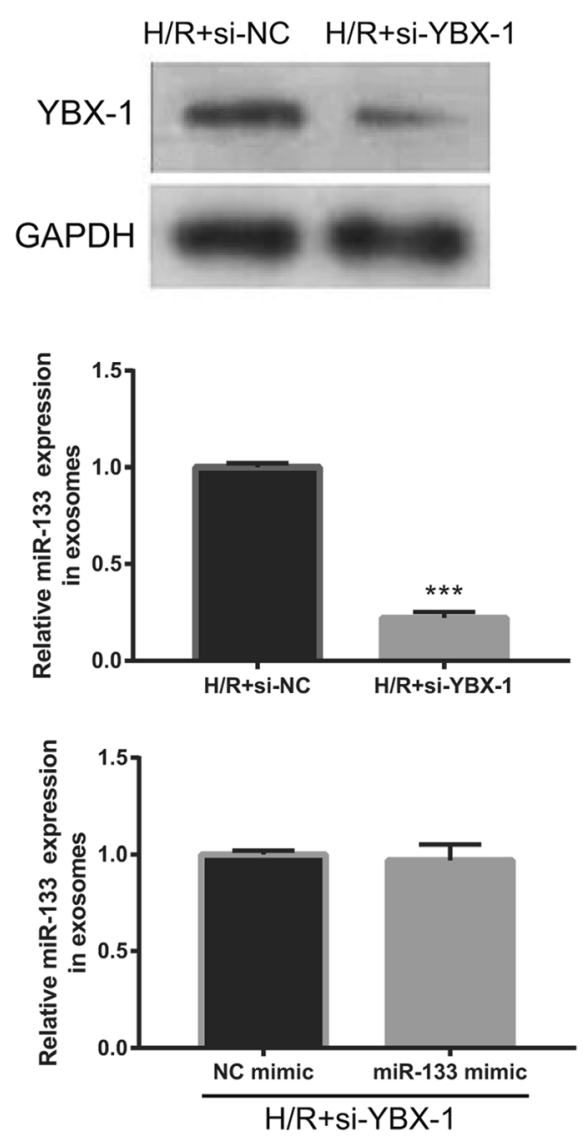

Fig. 7 Inhibition of specifically packaged miR-133 into EPC-derived exosomes by YBP1 silencing. a qRT-PCR and western blot analysis of YBX1 expression in H/R-induced EPCs at $48 \mathrm{~h}$ following si-YBX1 transfection. $\mathbf{b}$ qRT-PCR analysis of miR-133 expression in H/R-induced EPCs and exosomes at $48 \mathrm{~h}$ following si-YBX1 transfection. $\mathbf{c}$ qRT-PCR analysis of miR-133 expression in H/R-induced EPCs and exosomes at $48 \mathrm{~h}$ after co-transfection with si-YBX1 and miR-133 mimic 


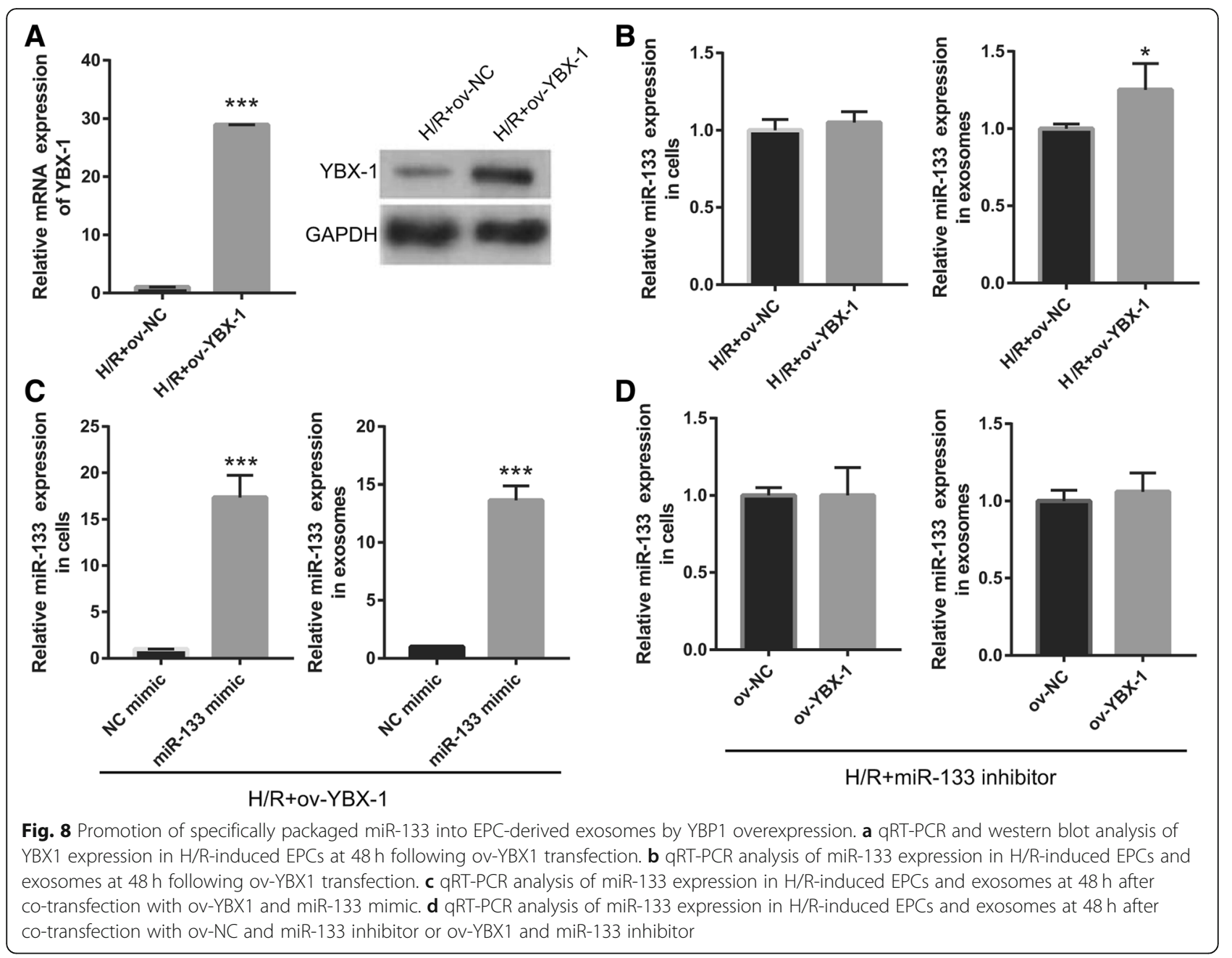

YBX-1 regulates fibroblast angiogenesis and MEndoT through exosome-transferred miR-133 regulation

We further examined whether YBX-1 could regulate miR-133 transfer to reduce fibroblast angiogenesis and MEndoT. First, miR-133 expression in fibroblasts treated with H/R-si-YBX1+miR-133 inhibitor/EPC-exosomes and H/R-ov-YBX1+miR-133 inhibitor/EPC-exosomes was significantly lower than that in fibroblasts treated with H/R-ov-YBX1/EPC-exosomes and H/R+ov-NC/ EPC-exosomes (Fig. 9a). The number of meshes had a similar trend of miR-133 expression in each group (Fig. 9b, c). Finally, endothelial markers CD31, VE-cadherin, and vWF expression levels in fibroblasts treated with H/R-si-YBX1+miR-133 inhibitor/EPC-exosomes and H/R-ov-YBX1+miR-133 inhibitor/EPC-exosomes were significantly lower than fibroblasts treated with $\mathrm{H} /$ R-ov-YBX1/EPC-exosomes and $\mathrm{H} / \mathrm{R}+\mathrm{ov}-\mathrm{NC} / \mathrm{EPC}$-exosomes. In contrast, fibrosis markers $\alpha$-SMA, N-cadherin, vimentin, and collagen I were significantly higher (Fig. 10). The results showed that fibroblasts treated with H/R-ov-YBX1/EPC-exosomes and H/R+ov-NC/EPC- exosomes had no significant effects on fibroblast angiogenesis and MEndoT. Fibroblast angiogenesis and MEndoT were significantly inhibited in fibroblasts treated with H/R-si-YBX1+miR-133 inhibitor/EPCexosomes and H/R-ov-YBX1+miR-133 inhibitor/ EPC-exosomes compared to fibroblasts treated with H/R-ov-YBX1/EPC-exosomes due to miR-133 silencing. The results suggested that YBX-1 knockdown inhibited miR-133 exosome transfer to reduce fibroblast angiogenesis and MEndoT.

\section{Discussion}

Cardiovascular disease induces fibroblast proliferation and excessive activation, resulting in cardiac fibrosis. Fibroblasts undergo MEndoT to obtain endothelial cell-like functions and participate in angiogenesis in the cardiac injury area, which can reverse myocardial fibrosis [4]. In a previous study, we found that H/REPC-exosomes increase angiogenesis in cardiac fibroblasts by promoting MEndoT. Hence, it is necessary to study the mechanism of H/R-EPC-exosomes to 

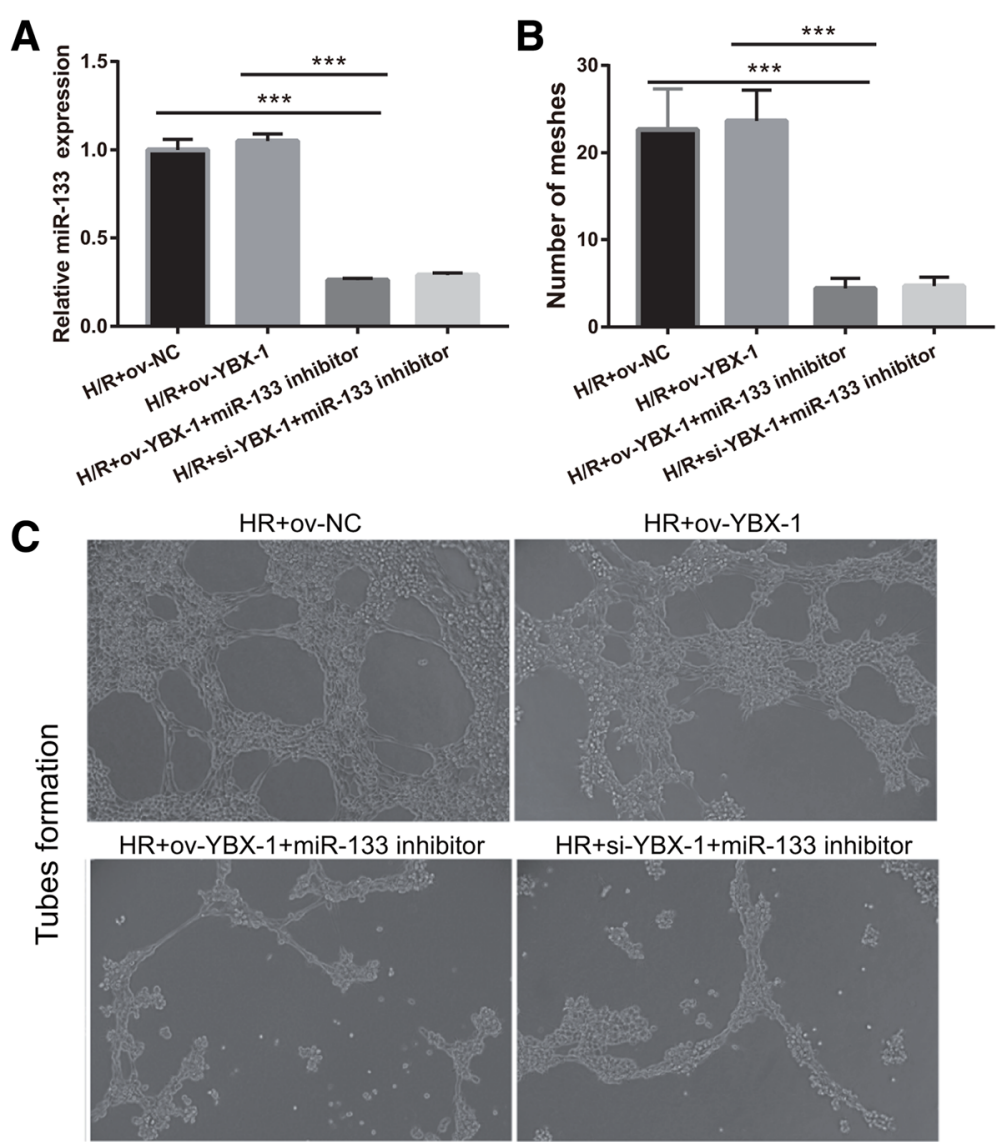

Fig. 9 Silencing of YBX1 in H/R-induced EPCs inhibits fibroblast angiogenesis. a miR-133 expression was measured by qRT-PCR in fibroblasts treated with H/R, H/R+ov-NC, H/R+si-YBX1+miR-133 inhibitor, and H/R+ ov-YBX1+miR-133 inhibitor-induced EPC-derived exosomes for $48 \mathrm{~h}$. b The bar graph represents quantification of the number of meshes per group. c Representative image of tube formation analysis. Data are shown as mean $\pm \mathrm{SD}$. ${ }^{* * *} P<0.001$

promote conversion of cardiac fibroblasts into endothelial cells and identify novel targets for cardiac fibrosis therapy. In this study, we found that $\mathrm{H} / \mathrm{R}-$ EPC-exosomes increased angiogenesis in cardiac fibroblasts by promoting MEndoT. Additionally, miR-133 was overexpressed in H/R-EPC-exosomes and functionally required for MEndoT of cardiac fibroblasts.

EPCs, a type of hematopoietic stem cell, promote vascular repair, including vascular proliferation and remodeling, when organs suffer from ischemic injury or endothelial damage $[22,23]$. EPCs have been identified to participate in the alleviation of cardiac fibrosis in several cardiovascular diseases [24, 25]. Several studies have described effective EPC-mediated attenuation of renal/fibrosis via exosomes [5, 26]. EPC-derived exosomes are complex particles formed by exocytosis of the EPC cell membrane, which can act as effective messengers for transmission of cell signaling and biological function between cells [5, 27]. Importantly, we found that H/R-induced EPC-derived exosomes increased angiogenesis in cardiac fibroblasts by promoting MEndoT [5]. Here, we studied the contribution of exosomal miRNAs in regulating the MEndoT of cardiac fibroblasts. Our results found that $H / R$ treatment promoted the senescence and apoptosis of EPCs. Wang et al. found that serum deprivation plus TNFa stimulation promoted EPC apoptosis and exosome release to improve H/R-induced endothelial dysfunction [28]. These results suggested that the onset of senescence or apoptosis may greatly affect the release of exosomes. Additionally, miR-133 was overexpressed in H/R-EPC-exosomes by a miRNA microarray assay and qRT-PCR verification. H/R-EPCexosome treatment inhibited promoted fibroblast angiogenesis and MEndoT. We also found that silencing of miR-133 expression in EPCs inhibited miR-133 expression in EPC-derived exosomes, which inhibited the intercellular transfer of miR-133 into fibroblasts and inhibited fibroblast angiogenesis and MEndoT. The results suggested that EPC-derived exosomes promoted fibroblast angiogenesis and MEndoT through the intercellular transfer of miR-133 thereby attenuating myocardial fibrosis. 


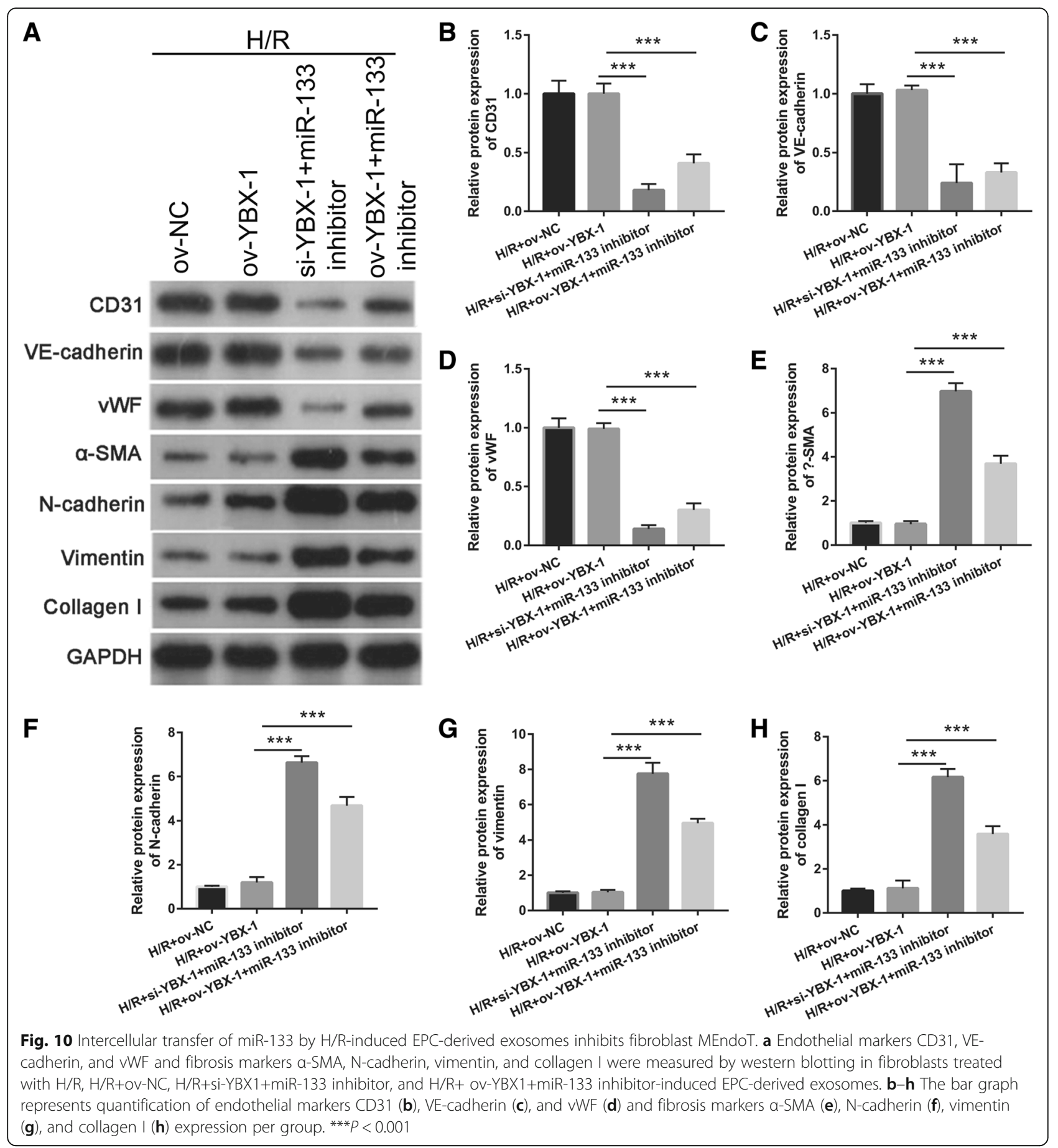

Studies have shown that the mechanism by which miRNAs are specifically loaded into exosomes is associated with specific RNA-binding proteins, such as YBX-1 [29], SYNCRIP [30, 31], and hnRNPA2B1 [32]. Here, we uncovered that YBX-1 expression was significantly enhanced in H/R-EPC as compared to SYNCRIP and hnRNPA2B1 expression. YBX1, which is localized to cytoplasmic granules, is an RNA-/DNA-binding multifunctional protein. The YBX1 protein is one of the most important transcriptional regulator proteins in exosomes [33]. Recently, some studies have found that Y box protein 1 is required to sort mRNAs, miRNAs, and lncRNA into exosomes [29, 34-36]. In this study, we found that YBX-1 upregulation in H/R-EPC did not affect miR-133 expression in $\mathrm{H} / \mathrm{R}$-EPC and H/R-EPC-exosomes. Additionally, YBX-1 upregulation did not affect fibroblast angiogenesis and MEndoT. Furthermore, we found that YBX-1 silencing did not affect miR-133 expression 
in H/R-EPC; however, it inhibited miR-133 expression in H/R-EPC-exosomes and prevented fibroblast angiogenesis and MEndoT. These results suggested that miR133 was specially sorted into H/R-EPC-exosomes via YBX-1. Further, YBX-1 silencing inhibited miR-133 transfer and reduced fibroblast angiogenesis and MEndoT.

\section{Conclusion}

miR-133 was specially sorted into H/R-EPC-exosomes via YBX-1 to increase fibroblast angiogenesis and MEndoT. Our findings suggest that miR-133 and YBX-1 are potential therapeutic targets to improve myocardial fibrosis.

\section{Abbreviations}

EPC: Endothelial progenitor cell; MEndoT: Mesenchymal-endothelial transition; H/R: Hypoxia/reoxygenation; YBX-1: Y box binding protein 1; H/REPC: H/R-induced EPC; H/R-EPC-exosomes: H/R-EPC-derived exosomes; Normal-EPC-exosomes: Normal cultured EPC-derived exosomes

\section{Acknowledgements}

None.

\section{Authors' contributions}

$\mathrm{FL}, \mathrm{XK}$, and $\mathrm{XH}$ designed and planned the study. $\mathrm{FL}, \mathrm{ZZ}, \mathrm{YS}$, and $\mathrm{LL}$ collected the data, and $F L, Z W, X Z$, and $Z L$ analyzed the data. FL was a major contributor in writing the manuscript. $\mathrm{XK}$ and $\mathrm{XH}$ amended the manuscript and provided the funds. All authors read and approved the final manuscript.

\section{Funding}

The study was supported by grants from the Natural Science Foundation of Guangdong Province of China (2018A030313771), the National Natural Science Foundation of China (NO.81700263), and the Science and Technology project of Shenzhen city of China (JCYJ20160426100250466 and JCYJ20170306152620264).

\section{Availability of data and materials}

The datasets generated and/or analyzed during the current study are available from the corresponding author on reasonable request.

\section{Ethics approval and consent to participate}

Not applicable.

\section{Consent for publication}

Not applicable.

\section{Competing interests}

The authors declare that they have no competing interests.

\section{Author details}

${ }^{1}$ Department of Cardiology, Shenzhen Bao'an Traditional Chinese Medicine Hospital Group, The Affiliated Hospital of Guangzhou University of Chinese Medicine, Shenzhen 518133, China. ${ }^{2}$ Graduate School, Guangzhou University of Chinese Medicine, Guangzhou 510405, China. ${ }^{3}$ Department of Cardiology, Fuwai Hospital, Chinese Academy of Medical Sciences, Shenzhen 518057, Guangdong, China. ${ }^{4}$ Shenzhen University School of Medicine \& Shenzhen University Health Science Center, No. 12, Langshan Road, Nanshan District, Shenzhen 518057, Guangdong, China. ${ }^{5}$ Department of Cardiology, The First Affiliated Hospital, Sun Yat-Sen University, Guangzhou 510080, Guangdong, China. ${ }^{6}$ Key Laboratory on Assisted Circulation, Ministry of Health, Guangzhou 510080, Guangdong, China.
Received: 16 April 2019 Revised: 24 July 2019

Accepted: 7 August 2019 Published online: 23 August 2019

\section{References}

1. Bittencourt MI, Cader SA, Araujo DV, Salles ALF, Albuquerque FN, Spineti PPM, Albuquerque DC, Mourilhe-Rocha R. Role of myocardial fibrosis in hypertrophic cardiomyopathy: a systematic review and updated metaanalysis of risk markers for sudden death. Arq Bras Cardiol. 2019;112:281-9.

2. Ning BB, Zhang Y, Wu DD, Cui JG, Liu L, Wang PW, Wang WJ, Zhu WL, Chen $Y$, Zhang T. Luteolin-7-diglucuronide attenuates isoproterenol-induced myocardial injury and fibrosis in mice. Acta Pharmacol Sin. 2017;38:331-41.

3. Wary A, Wary N, Baruah J, Mastej V, Wary KK. Chromatin-modifying agents convert fibroblasts to OCT4+ and VEGFR-2+ capillary tube-forming cells. PLoS One. 2017;12:e0176496.

4. Ubil E, Duan J, Pillai IC, Rosa-Garrido M, Wu Y, Bargiacchi F, Lu Y, Stanbouly S, Huang J, Rojas M, et al. Mesenchymal-endothelial transition contributes to cardiac neovascularization. Nature. 2014;514:585-90.

5. Ke X, Yang D, Liang J, Wang X, Wu S, Wang X, Hu C. Human endothelial progenitor cell-derived exosomes increase proliferation and angiogenesis in cardiac fibroblasts by promoting the mesenchymal-endothelial transition and reducing high mobility group box 1 protein B1 expression. DNA Cell Biol. 2017:36:1018-28.

6. Raposo G, Stoorvogel W. Extracellular vesicles: exosomes, microvesicles, and friends. J Cell Biol. 2013;200:373-83.

7. Gu S, Zhang W, Chen J, Ma R, Xiao X, Ma X, Yao Z, Chen Y. EPC-derived microvesicles protect cardiomyocytes from Ang II-induced hypertrophy and apoptosis. PLoS One. 2014;9:e85396.

8. Ranghino A, Cantaluppi V, Grange C, Vitillo L, Fop F, Biancone L, Deregibus MC, Tetta C, Segoloni GP, Camussi G. Endothelial progenitor cell-derived microvesicles improve neovascularization in a murine model of hindlimb ischemia. Int J Immunopathol Pharmacol. 2012;25:75-85.

9. Hayakawa K, Chan SJ, Mandeville ET, Park JH, Bruzzese M, Montaner J, Arai K, Rosell A, Lo EH. Protective effects of endothelial progenitor cell-derived extracellular mitochondria in brain endothelium. Stem Cells. 2018;36:1404-10.

10. Yu X, Odenthal M, Fries JW. Exosomes as miRNA carriers: formationfunction-future. Int J Mol Sci. 2016;17.

11. Fleshner M, Crane CR. Exosomes, DAMPs and miRNA: features of stress physiology and immune homeostasis. Trends Immunol. 2017;38:768-76.

12. Zhou Y, Li P, Goodwin AJ, Cook JA, Halushka PV, Chang E, Fan H. Exosomes from endothelial progenitor cells improve the outcome of a murine model of sepsis. Mol Ther. 2018;26:1375-84.

13. Zhou Y, Li P, Goodwin AJ, Cook JA, Halushka PV, Chang E, Zingarelli B, Fan $H$. Exosomes from endothelial progenitor cells improve outcomes of the lipopolysaccharide-induced acute lung injury. Crit Care. 2019;23:44.

14. Thum T. Noncoding RNAs and myocardial fibrosis. Nat Rev Cardiol. 2014:11:655-63.

15. Tao H, Yang JJ, Hu W, Shi KH, Deng ZY, Li J. Noncoding RNA as regulators of cardiac fibrosis: current insight and the road ahead. Pflugers Arch. 2016:468:1103-11.

16. Creemers EE, van Rooij E. Function and therapeutic potential of noncoding RNAs in cardiac fibrosis. Circ Res. 2016;118:108-18.

17. Chen P, Pan J, Zhang X, Shi Z, Yang X. The role of microRNA-181a in myocardial fibrosis following myocardial infarction in a rat model. Med Sci Monit. 2018;24:4121-7.

18. Verjans $R$, Peters $T$, Beaumont FJ, van Leeuwen $R$, van Herwaarden $T$, Verhesen W, Munts C, Bijnen M, Henkens M, Diez J, et al. MicroRNA-221/222 family counteracts myocardial fibrosis in pressure overload-induced heart failure. Hypertension. 2018;71:280-8.

19. Yuan J, Liu H, Gao W, Zhang L, Ye Y, Yuan L, Ding Z, Wu J, Kang L, Zhang X, et al. MicroRNA-378 suppresses myocardial fibrosis through a paracrine mechanism at the early stage of cardiac hypertrophy following mechanical stress. Theranostics. 2018;8:2565-82.

20. Nie X, Fan J, Li H, Yin Z, Zhao Y, Dai B, Dong N, Chen C, Wang DW. miR-217 promotes cardiac hypertrophy and dysfunction by targeting PTEN. Mol Ther Nucleic Acids. 2018;12:254-66.

21. Yang Z, Wu L, Zhu X, Xu J, Jin R, Li G, Wu F. MiR-29a modulates the angiogenic properties of human endothelial cells. Biochem Biophys Res Commun. 2013:434:143-149.

22. Wils J, Favre J, Bellien J. Modulating putative endothelial progenitor cells for the treatment of endothelial dysfunction and cardiovascular complications in diabetes. Pharmacol Ther. 2017;170:98-115. 
23. Asahara T, Murohara T, Sullivan A, Silver M, van der Zee R, Li T, Witzenbichler B, Schatteman G, Isner JM. Isolation of putative progenitor endothelial cells for angiogenesis. Science. 1997;275:964-7.

24. Yuan Z, Kang L, Wang Z, Chen A, Zhao Q, Li H. 17beta-estradiol promotes recovery after myocardial infarction by enhancing homing and angiogenic capacity of bone marrow-derived endothelial progenitor cells through ERalpha-SDF-1/CXCR4 crosstalking. Acta Biochim Biophys Sin Shanghai. 2018:50:1247-56.

25. Mudyanadzo TA. Endothelial progenitor cells and cardiovascular correlates. Cureus. 2018;10:e3342.

26. Yang J, Wang M, Zhu F, Sun J, Xu H, Chong Lee Shin OL, Zhao Z, Pei G, Zhu $\mathrm{H}, \mathrm{CaO} \mathrm{C}$, et al. Putative endothelial progenitor cells do not promote vascular repair but attenuate pericyte-myofibroblast transition in UUOinduced renal fibrosis. Stem Cell Res Ther. 2019;10:104.

27. Tkach $\mathrm{M}$, Thery C. Communication by extracellular vesicles: where we are and where we need to go. Cell. 2016;164:1226-32.

28. Wang J, Chen S, Ma X, Cheng C, Xiao X, Chen J, Liu S, Zhao B, Chen Y. Effects of endothelial progenitor cell-derived microvesicles on hypoxia/ reoxygenation-induced endothelial dysfunction and apoptosis. Oxidative Med Cell Longev. 2013;2013:572729.

29. Shurtleff MJ, Temoche-Diaz MM, Karfilis KV, Ri S, Schekman R. Y-box protein 1 is required to sort microRNAs into exosomes in cells and in a cell-free reaction. Elife. 2016;5.

30. Hobor F, Dallmann A, Ball NJ, Cicchini C, Battistelli C, Ogrodowicz RW Christodoulou E, Martin SR, Castello A, Tripodi M, et al. A cryptic RNAbinding domain mediates Syncrip recognition and exosomal partitioning of miRNA targets. Nat Commun. 2018;9:831.

31. Santangelo L, Giurato G, Cicchini C, Montaldo C, Mancone C, Tarallo R, Battistelli C, Alonzi T, Weisz A, Tripodi M. The RNA-binding protein SYNCRIP is a component of the hepatocyte exosomal machinery controlling microRNA sorting. Cell Rep. 2016;17:799-808.

32. Qu L, Ding J, Chen C, Wu ZJ, Liu B, Gao Y, Chen W, Liu F, Sun W, Li XF, et al. Exosome-transmitted IncARSR promotes sunitinib resistance in renal cancer by acting as a competing endogenous RNA. Cancer Cell. 2016;29:653-68.

33. Ung TH, Madsen HJ, Hellwinkel JE, Lencioni AM, Graner MW. Exosome proteomics reveals transcriptional regulator proteins with potential to mediate downstream pathways. Cancer Sci. 2014:105:1384-92.

34. Kossinova OA, Gopanenko AV, Tamkovich SN, Krasheninina OA, Tupikin AE, Kiseleva E, Yanshina DD, Malygin AA, Ven'yaminova AG, Kabilov MR, Karpova GG. Cytosolic YB-1 and NSUN2 are the only proteins recognizing specific motifs present in mRNAs enriched in exosomes. Biochim Biophys Acta Proteins Proteom. 2017;1865:664-73.

35. Suresh PS, Tsutsumi R, Venkatesh T. YBX1 at the crossroads of non-coding transcriptome, exosomal, and cytoplasmic granular signaling. Eur J Cell Biol. 2018:97:163-67.

36. Shurtleff MJ, Yao J, Qin Y, Nottingham RM, Temoche-Diaz MM, Schekman R, Lambowitz AM. Broad role for YBX1 in defining the small noncoding RNA composition of exosomes. Proc Natl Acad Sci U S A. 2017;114:e8987-e8995.

\section{Publisher's Note}

Springer Nature remains neutral with regard to jurisdictional claims in published maps and institutional affiliations.

Ready to submit your research? Choose BMC and benefit from:
- fast, convenient online submission
- thorough peer review by experienced researchers in your field
- rapid publication on acceptance
- support for research data, including large and complex data types
- gold Open Access which fosters wider collaboration and increased citations
- maximum visibility for your research: over 100M website views per year
At BMC, research is always in progress.
Learn more biomedcentral.com/submissions

